\title{
25 Research Square \\ T Cell Exhaustion and Senescence in Epstein-Barr Virus-Associated Lymphoma Patients
}

Huihui Liu

Peking University First Hospital

Junhui Xu

Peking University First Hospital

Lihong Wang

Peking University First Hospital

Wenjun Mao

Peking University First Hospital

\section{Bingjie Wang}

Peking University First Hospital

\section{Zeyin Liang}

Peking University First Hospital

Yang Zhang

Peking University First Hospital

\section{Yongjin Shi}

Peking University First Hospital

\section{Bo Tang}

Peking University First Hospital

\section{Ye Shen}

Peking University First Hospital

\section{Yujun Dong}

Peking University First Hospital

\section{Hanyun Ren}

Peking University First Hospital

Xinan Cen ( $\nabla$ cenxn@bjmu.edu.cn )

\section{Research article}

Keywords: Epstein-Barr Virus, Epstein-Barr Virus-Associated Lymphoma, T cell, exhaustion, senescence

Posted Date: April 27th, 2020

DOI: https://doi.org/10.21203/rs.3.rs-23447/v1 
License: (c) (i) This work is licensed under a Creative Commons Attribution 4.0 International License. Read Full License 


\section{Abstract \\ Background}

The Epstein-Barr Virus (EBV) is tumorigenic, and can be detected in many kinds of lymphomas. Some studies have shown a worse prognosis for patients with EBV-associated lymphoma. However, the mechanism is not fully understood. This study aimed to investigate the T cell signatures in patients with EBV-associated lymphoma.

\section{Methods}

Peripheral blood was collected from 17 patients with EBV-associated lymphoma and 19 healthy donors. We first examined the proportions of the lymphocyte subpopulations in peripheral blood mononuclear cells in patients with both groups by flow cytometry. Then we employed the enzyme-linked immunospot assay to evaluate the EBV antigen-specific response of the cytotoxic T cells in the two groups. Finally, to explore the mechanism of T cells dysfunction in EBV-associated lymphoma, we examined the expression of multiple inhibitory receptors representing $T$ cell exhaustion and biomarkers representing $T$ cell senescence on the surfaces of $\mathrm{CD} 4^{+} \mathrm{T}$ cells and $\mathrm{CD} 8^{+} \mathrm{T}$ cells.

\section{Results}

The ratio of peripheral $\mathrm{CD} 4^{+} \mathrm{T}$ cells and the absolute cell counts of $\mathrm{CD} 4^{+} \mathrm{T}$ cells and $\mathrm{CD} 8^{+} \mathrm{T}$ cells were significantly decreased in patients with EBV-associated lymphoma compared with those of healthy donors. The IFN-y production upon stimulation of EBV mixed peptides were remarkably reduced in the patients. Higher expression levels of T cell exhaustion markers, PD1, LAG3, TIM3 and CTLA4 on T cells were found in the patients. The two subsects of exhausted T cells (T-bet ${ }^{\text {hi } P D} 1^{\text {mid }}$ and EOMES ${ }^{\text {hi } P D} 1^{\text {hi }}$ ) were higher in the patients. More importantly, CXCR5 ${ }^{+} \mathrm{CD} 8^{+} \mathrm{T}$ cells controlling viral replication decreased significantly in the patients. The fractions of senescent $T$ cells increased in the patients.

\section{Conclusions}

In summary, our study demonstrated that the reduced EBV-specific T cells, the exhaustion and senescence of $T$ cells together contributed to the T cell dysfunction in the patients with EBV-associated lymphoma.

\section{Introduction}

The tumorigenic Epstein-Barr Virus (EBV) has been linked to certain types of lymphoma such as Extranodal NK/T-cell lymphoma, Burkitt lymphoma, Angioimmunoblast T-cell lymphoma, Hodgkin's 
lymphoma and Post-transplant lymphoproliferative disease[1, 2]. These EBV positive lymphomas, also known as EBV-associated lymphomas, were found to have poorer prognosis [3-5]. The North American study group found that the plasma EBV-DNA levels before treatment and at 6 months post treatment were closely related to the prognosis of Hodgkin's lymphoma[3]. The French GELA study group found that EBV positive was an independent poor prognostic factor for peripheral T-cell lymphoma by univariate and multivariate analysis[4]. Previous work by our team on 263 patients with non-Hodgkin's lymphoma demonstrated a shorter median survival time and lower overall survival rate in patients with EBV-positive than those with EBV-negative[5]. Multivariate analysis also confirmed the peripheral blood EBV positive as an independent poor prognostic factor for lymphoma.

Controlling EBV infection requires the host immune system response, and a large number of genetic mutations affecting the function of various components of the immune system have been found to result in inadequate control of EBV infection[6, 7]. EBV specific T cells are particularly important for preventing and treating EBV-induced lymphoproliferative disease. Previous bone marrow transplant research found that donor-derived EBV-specific T lymphocytes can treat EBV-induced lymphoproliferative disorders in recipients[8]. Some malignant neoplasms associated with EBV, including gastric cancer, Hodgkin's lymphoma, and nasopharyngeal carcinoma, are typically infiltrated by a large number of inflammatory cells without tumor killing[9-13]. The phenotype and function of these host immune cells and whether these host immune cells surrounding the EBV-positive tumor cells can kill tumor cells under certain conditions are still unclear. Understanding the phenotype and function of host immune cell surrounding these tumor cells, we may be able to find treatments that alter the function of these host immune cells to improve the ability to kill tumor cells.

Dysfunctional T cells observed in cancer can be classified as anergic, exhausted or senescent based on phenotypic characteristics[14]. Anergy is a long-term stable state of T lymphocyte with low IL-2 production or incomplete activation, to which naive T cells fall upon low co-stimulatory and/or high coinhibitory stimulation[15]. T cell exhaustion were found in human carcinomas of lung, ovary, colon and melanomas[16-20]. Exhausted T cells are characterized as effector T cells with decreased cytokine secretion and effector function, and being resistant to reactivation[21]. T cell exhaustion is manifested by a significant high expression of various inhibitory receptors including PD1, CTLA4, Tim3 and LAG3 on the cell surface, as well as the abnormal expression of certain transcription factors such as Emoes and T-bet in the case of chronic antigen over-stimulation[22-24]. It is well acknowledged that T cell exhaustion plays an important role in the pathogenesis of various solid tumors, including melanoma, non-small-cell lung cancer, renal cancer, urothelial cancer, and head and neck squamous cell cancer[21]. More and more studies demonstrated the phenomenon of T cell exhaustion in hematological tumors, including certain special types of lymphoma, acute myelogenous leukemia, acute lymphocytic leukemia, chronic lymphocytic leukemia, adult T-cell leukemia/lymphoma and multiple myeloma[25-27]. Checkpoint inhibitors can partially reverse the $\mathrm{T}$ cell exhaustion, and have been applied to treat the malignant tumor diseases with the treatment efficiency fluctuating between 15-34\%[24]. The senescent T cells which are late-stage memory $T$ cells usually lack the expression of CD28, accompanied by the high expression of 
CD57 and KLRG-1[14]. This deterioration of immune function of $T$ cells contributes to the increased incidence among the elderly of morbidity and mortality from cancer[28].

T cell dysfunction can reduce the body's immune surveillance, leading to tumor cell escape, and malignant tumor progression[24]. The mechanisms of the pathogenesis of EBV-associated lymphoma is not fully elucidated. The role of the T cell dysfunction in the pathogenesis of EBV-associated lymphoma has not been studied.

\section{Materials And Methods Patients and healthy donors}

The study was approved by the Ethics Committee of Peking University First Hospital (Beijing, China; approval no. 2016[1033]). Informed consent was obtained from all individual participants included in the study. Peripheral blood samples were collected from 17 EBV-associated lymphoma patients and 19 healthy volunteers attending our clinic. These patients have many pathological types. The clinical characteristics of the patients and healthy volunteers are shown in Table 1.

\section{ELISPOT}

Peripheral blood mononuclear cells (PBMC) were obtained by Ficoll (HAO YANG BIOLOGICAL MANUFACTURE CO., Tianjin, China). EBV mix peptide $(10 \mu \mathrm{g} / \mathrm{ml}$, thinkpeptides) or DMSO controls diluted in complete RPMI-1640 were added to appropriate wells at $50 \mu \mathrm{l} /$ well, and then PBMC $\left(2 \times 10^{6} / \mathrm{mL}\right)$ at $50 \mu$ /well were added and incubated at $37^{\circ} \mathrm{C}$, in a $5 \% \mathrm{CO}_{2}$ humidified incubator for 24 hours. ELISPOT analysis was performed by Human IFN-y precoated ELISPOT kit (DAKEWE, Shenzhen, China).

\section{Flow cytometric analysis and Phenotypic analysis of T cell}

Flow cytometry was performed using the following anti-mouse antibodies: PD1-FITC, PD1-PE/cy7, LAG3PE, CD4-PerCP/Cy5.5, KLRG1-APC, CD8-PE/cy7, CD8-APC/cy7, CD28-APC, T-bet-APC, CXCR5-APC (Biolegend, Cal., US); CD3-FITC,CD3-PerCP/Cy5.5ロCD57-FITC, CD8-PE, CTLA4-PE, Tim3-APC, CD56-FITC, CD56-APC/cy7, CD19-APC/cy7, CD45-BV421 (BD Biosciences, NJ, US); Eomes-FITC (eBioscience, Cal., US). Flow cytometry analysis was performed on FACS Canto II (BD Biosciences, NJ, US) and analyzed with FlowJo software.

\section{Statistical Analysis}

The data are expressed as the mean \pm SEM and tested for statistical significance with Student's t-test using GraphPad Prism 5 software. ${ }^{*} p<0.05,{ }^{*} p<0.01$, and $* \star * p<0.001$.

\section{Results}




\section{The absolute counts of lymphocyte subpopulation decreased in EBV-associated lymphoma patients}

We first examined the proportions of the lymphocyte subpopulations in peripheral blood mononuclear cells in patients with EBV-associated lymphoma and healthy donors by flow cytometry. The proportions of $\mathrm{CD} 4^{+} \mathrm{T}$ cells and $\mathrm{B}$ cells in $\mathrm{CD} 45^{+}$cells were significantly decreased in the patients than in healthy donors (Fig. 1A). There were no obvious differences of the proportions of peripheral $\mathrm{CD}^{+} \mathrm{T}$ cells, $\mathrm{CD} 8^{+} \mathrm{T}$ cells and NK cells in patients with EBV-associated lymphoma and healthy donors (Fig. 1A). We then compared the absolute cell counts of the lymphocyte subpopulations from peripheral blood. We found the absolute counts of all lymphocyte subpopulations, including $\mathrm{CD}^{+} \mathrm{T}$ cells, $\mathrm{CD} 4^{+} \mathrm{T}$ cells, $\mathrm{CD} 8^{+} \mathrm{T}$ cells, NK cells and B cells, were significantly decreased in peripheral blood of patients with EBV-associated lymphoma than those of healthy donors (Fig. 1B).

\section{EBV antigen-specific cytotoxic T cells decreased in EBV- associated lymphoma}

Given the significantly decreased absolute $T$ cell counts in peripheral blood of patients with EBVassociated lymphoma, we wonder whether the function of the T cell are also impaired. We employed the enzyme-linked immunospot assay to evaluate the EBV antigen-specific response of the cytotoxic $T$ cells in the two groups. We examined the IFN- $y$ secretion in cytotoxic T cells upon stimulation of mixed EBV peptides. We observed a large number of EBV antigen-specific T cells (the diffusely distributed spots under the microscope) in healthy donors(Figure 2A), while a small amount of EBV antigen-specific T cells (the sporadic spots) in patients with EBV-associated lymphomaःFigure 2B). We counted the number of spots and found significantly more EBV antigen-specific cytotoxic T cells in the peripheral blood of healthy donors than that of patients with EBV-associated lymphoma (Fig. 2C).

\section{T cell exhaustion in patients with EBV-associated lymphoma}

To explore the mechanism of T cells dysfunction in EBV-associated lymphoma, we examined the expression of multiple inhibitory receptors representing $T$ cell exhaustion on the surfaces of $\mathrm{CD} 4^{+} \mathrm{T}$ cells and $\mathrm{CD} 8^{+} \mathrm{T}$ cells. We found the proportions of PD1, CTLA4, TIM3 and LAG3-expressing $\mathrm{CD} 4^{+}$and $\mathrm{CD} 8^{+} \mathrm{T}$ cells were higher significantly in the lymphoma patients than in healthy donors (Fig. 3A-D). We further examined the proportion of the two different types of exhausted T cells: the T-bet ${ }^{\text {hi }}$ PD1 ${ }^{\text {mid }}$ cells, which can be rescued by blockade of PD1 pathway, and the EOMES ${ }^{\text {hi } P D} 1$ hi cells, which cannot be rescued by the PD1 pathway blockade. Higher proportions of both types of exhausted $\mathrm{CD} 4^{+} \mathrm{T}$ and $\mathrm{CD} 8^{+} \mathrm{T}$ cells were observed in patients with EBV-associated lymphoma than in healthy donors (Fig. 3E-F). More importantly, a subset of exhausted CD8 ${ }^{+} T$ cells expressing the chemokine receptor CXCR5 plays a key role in the 
control of viral replication[29]. We found the proportion of CXCR5 ${ }^{+} \mathrm{CD} 8^{+} \mathrm{T}$ cells were decreased significantly in the patients (Fig. 3G).

\section{T cell senescence in patients with EBV-associated lymphoma}

To evaluate the senescence state of the T cells, we examined the expression of the surface markers CD28, CD57 and KLRG- 1 on CD $4^{+}$and CD $8^{+}$T cells in patients with EBV-associated lymphoma and healthy donors. We discovered a significant reduction in the percentage of $\mathrm{CD} 28^{+}$cells in both $\mathrm{CD} 4^{+}$and $\mathrm{CD}^{+} \mathrm{T}$ cells in patients with EBV-associated lymphoma (Fig. 4A), while a significant increase in $\mathrm{CD} 57^{+}$ cells (Fig. 4B). In addition, we found a significant increased proportion for KLRG-1-expressing cells in CD4 ${ }^{+}$and $\mathrm{CD} 8^{+} \mathrm{T}$ cells (Fig. $4 \mathrm{C}$ ).

\section{Discussion}

In this study, we investigated the T cell dysfunction in EBV associated lymphoma by comparing to healthy donors. We observed that EBV-specific cytotoxic $T$ cells reduced significantly in patients than in healthy donors and displayed features of exhaustion and senescence.

Exhausted T cells usually express higher level of inhibitory receptors such as PD1, CTLA4, LAG3 and Tim3[22-24]. PD1 is a member of the B7 receptor family which plays an important role in the immune response, and transmits regulatory signals to inhibit $\mathrm{T}$ cell activation and proliferation, thereby mediating immune escape of tumor cells[30]. EBV-infected lymphoma cells have been reported to express PD-L1, a ligand for PD1[31, 32]. High expression of PD1 on the surfaces of $C D 4^{+} T$ and $C D 8^{+} T$ were found in the humanized mouse models of EBV-positive DLBCL (diffuse large B-cell lymphoma) and the peripheral blood of EBV-positive DLBCL patients[33, 34]. In a humanized mouse model, PD-1 blockade greatly enhanced the ability of T cells to produce the anti-tumor cytokine IFN- $\gamma$ in response to EBV peptides and decreased the growth of EBV-induced lymphomas[33]. Our results also showed higher expression of the inhibitory receptors, especially PD1, CTLA4, Tim3 and LAG3 on peripheral CD4 ${ }^{+}$and $\mathrm{CD} 8^{+} \mathrm{T}$ cells in the patients with EBV-associated lymphoma than healthy donors, suggesting $T$ cells exhaustion. Furthermore, our study suggested that $T$ cell exhaustion existed widely across different types of lymphoma, including not only EBV-positive diffuse large B-cell lymphoma, but also EBV-associated Extranodal NK/T-cell lymphoma, Angioimmunoblast T-cell lymphoma and Hodgkin's lymphoma.

Several studies found a variety of transcription factors including Eomes, T-bet, Blimp-1, BATF, Fox01 andVHL were expressed in exhausted T cells[22].T-bet could decrease the expression of inhibitory receptors during chronic viral infection, resulting in more slightly exhausted $\mathrm{CD} 8^{+} \mathrm{T}$ cells[35]. The transcription factors T-bet and EOMES together define and maintain exhausted T cell subsets both in mice and humans. Two distinct types of exhausted T cells exist, including the progenitor and terminal subsets, which is confirmed in both mouse and human[35]. The progenitor subset featuring T- 
bet ${ }^{\text {hi }}$ PD $1{ }^{\text {mid }}$. has strong proliferative capacity and can be transformed into a terminal subset with the chronic stimulation of antigen[35,36]. The terminal subset with the phenotype of EOMES ${ }^{\text {hiPD }} 1^{\text {hi }}$ express higher levels of inhibitory receptors with reducing co-production of antiviral cytokines[36]. The Tbet ${ }^{\text {hi }}$ PD $1^{\text {mid }}$ exhausted T cells can be rescued by blockade of PD1 pathway while the EOMES ${ }^{\text {hi } P D} 1^{\text {hi }}$ exhausted T cells cannot. In this study, we found higher proportion of exhausted CD $4^{+}$and $\mathrm{CD} 8^{+} \mathrm{T}$ cells of both phenotypes in patients with EBV-associated lymphoma than in healthy donors.

Checkpoint inhibitors can partially reverse the T cell exhaustion of malignant tumors and have been applied in these malignant tumor diseases treatment[24]. PD1 inhibitors have good therapeutic effect in a variety of lymphomas, especially in Hodgkin's lymphoma with a slightly higher EBV-positive rate[37].Dramatic responses to pembrolizumab (PD1 blockade) were observed with an overall response rate of $100 \%$ in EBV-positive metastatic gastric cancer[38].These phenomena illustrate that EBV may also have an aggravating effect on T cell exhaustion. Therefore, immunological checkpoint inhibitor may achieve certain efficacy in patients with EBV-associated lymphoma. Our study found that peripheral blood $T$ cells from patients with EBV-associated lymphoma expressed multiple inhibitory receptors simultaneously, including PD1, CTLA4, LAG3, TIM3, and had both types of exhausted T cells (Tbet $^{\text {hi}}$ PD $1^{\text {mid }}$ and EOMES ${ }^{\text {hi } P D 1} 1^{\text {hi }}$ exhausted T cells). Previous studies have found that the ability to inhibit tumor growth using PD1 blockade in combination with CTLA4 blockade is stronger than one of the checkpoint inhibitors alone in NSG mice with EBV-positive DLBCL[33]. CTLA4 blockade could increase the frequency of IFN- $y$ and granzyme-B expressing $C D 8^{+} T$ cells independently of T-bet by selectively inhibiting the accumulation of Eomesodermin mRNA and protein[38]. The EOMES ${ }^{\text {hiPD }} 1^{\text {hi }}$ exhausted T cells may be rescued by CTLA4 blockade which compensate for the killing effect of PD1 blockade on the above two subsets of exhausted T cells. Using multiple immune checkpoint inhibitors to treat EBVassociated lymphoma may more completely reverse $T$ cell exhaustion and achieve better efficacy.

A study defined that a subset of exhausted $\mathrm{CXCR} 5^{+} \mathrm{CD} 8^{+} \mathrm{T}$ cells plays an important role in the control of

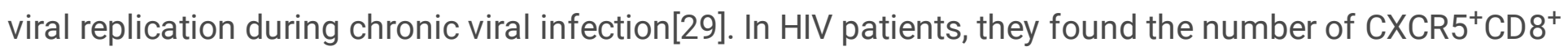
T cells was inversely correlated with viral load. The $\mathrm{CXCR} 5^{+}$population displayed better treatment than the $\mathrm{CXCR}^{-}$population when adoptively transferred to chronically infected mice and showed the synergistic result on decreasing viral load when combined with anti-PD-L1 treatment[29]. In this study, we found the $\mathrm{CXCR}^{+} \mathrm{CD}^{+} \mathrm{T}$ cells reduced in EBV-associated lymphoma patients, suggesting the anti-viral immune response was weakened in EBV-associated lymphoma.

Senescence and exhausted T cells do have some similarity in certain aspects of functionality but they are not entirely the same[14,39]. Low expression of CD28 and high expression of CD57 and KLRG-1 are considered to be the key markers that distinguish T cell senescence from T cell exhaustion[40, 41]. Some studies have demonstrated that exhausted T cells do not derive from the "senescent" KLRG-1+ effector T cells but from the memory precursors in the effector phase[40, 41]. In our study, we found that CD28 expression was decreased, KLRG-1and CD57 expression were significantly increased in peripheral blood 
$\mathrm{CD} 4^{+} \mathrm{T}$ cells and $\mathrm{CD} 8^{+} \mathrm{T}$ cells in patients with EBV-associated lymphoma. Therefore, $\mathrm{T}$ cell senescence might be another contributor of $\mathrm{T}$ cell dysfunction in the patients with EBV-associated lymphoma.

More EBV antigen-specific cytotoxic T cells in healthy donors were found in healthy donors than in patients with EBV-associated lymphoma, suggesting insufficient EBV specific T responses in patients. Donor lymphocyte infusion can not only increase the recipient's EBV antigen-specific T lymphocytes directly, but also reverse the T cell exhaustion to promote the tumor-killing ability of the immune system[8, 42]. Donor lymphocyte infusion has been used in the treatment of EBV-associated lymphoma and has achieved good efficacy[43, 44]. Our study found less EBV antigen-specific T lymphocytes and T cell exhaustion in patients with EBV-associated lymphoma, suggesting donor lymphocyte infusion could be a good treatment strategy of EBV-associated lymphoma.

\section{Conclusion}

In summary, T cell dysfunction was the predominant feature of the patients with EBV-associated lymphoma, including reducing EBV-specific cytotoxic $T$ cells, $T$ cell exhaustion and $T$ cell senescence. Immune checkpoint inhibitors and donor lymphocyte infusion may be effective treatments for EBVassociated lymphoma.

\section{Abbreviations}

EBV:Epstein-BarrVirus; IFN-ץ:Interferon-ү; PD1:Programmed cell death protein 1; LAG3:Lymphocyteactivation gene 3; TIM3:T cell immunoglobulin and mucin domain-containing protein 3;CTLA4:Cytotoxic T-Lymphocyte-Associated Antigen 4; T-bet:T-box expressed in T cells; EOMES:Eomesodermin; CXCR5:C-XC chemokine receptor type 5;IL-2:Interleukin-2; KLRG-1:Killer cell lectin-like receptor G1; ELISOPT:enzyme linked immunospot assay; PBMC:Peripheral blood mononuclear cells;PD-L1:Programmed cell death 1 ligand 1; DLBCL:Diffuse large B-cell lymphoma; Blimp-1:B lymphocyte induced maturation protein 1; BATF:Basic leucine zipper transcriptional factor ATF-like; Fox01:Forkhead box 01; VHL:Von HippelLindau.

\section{Declarations}

\section{Availability of data and materials}

Not applicable.

\section{Ethics approval and consent to participate}

The Ethics Committee of Peking University First Hospital provided ethical approval, and informed consent for collecting and preserving samples and details was obtained from each patient. 


\section{Consent for publication}

Not applicable.

\section{Author details}

Department of Hematology, Peking University First Hospital, Beijing, 100010, China

\section{Competing interests}

The authors declare that they have no competing interests.

\section{Funding}

This work was supported by National Natural Science Foundation of China (No. 81600144),Beijing Municipal Science Technology Commission( No. Z191100006619026 and Z141107002514017).

\section{Authors' contributions}

HHL performed ELISPOT, flow cytometric analysis and phenotypic analysis of T cell, statistical analysis, and wrote the paper; JHX collected the peripheral blood samples, statistical analysisand helped write the paper; LHW performed flow cytometric analysis and phenotypic analysis of T cell; XNC designed this project and supervised this project; WM, BJW, ZYL, YZ, YJS, BT, YS, YJD and HYRprovided technical supportand clinical data. All authors read and approved the final manuscript.

\section{Acknowledgments}

Not applicable.

\section{References}

1. Williams $\mathrm{H}$, Crawford $\mathrm{DH}$. Epstein-Barr virus: the impact of scientific advances on clinical practice. Blood. 2006;107:862-9.

2. Dierickx D, Habermann TM. Post-Transplantation Lymphoproliferative Disorders in Adults. N Engl J Med. 2018;378:549-62.

3. Kanakry JA, Li H, Gellert LL, Lemas MV, Hsieh WS, Hong F, et al. Plasma Epstein-Barr virus DNA predicts outcome in advanced Hodgkin lymphoma: correlative analysis from a large North American cooperative group trial. Blood. 2013;121:3547-53. 
4. Dupuis J, Emile JF, Mounier N, Gisselbrecht C, Martin-Garcia N, Petrella T, et al. Prognostic significance of Epstein-Barr virus in nodal peripheral T-cell lymphoma, unspecified: A Groupe d'Etude des Lymphomes de l'Adulte (GELA) study. Blood. 2006;108:4163-9.

5. Wang B, Cen X, Ren H, Liu W, Liang Z, Qiu Z, et al. Epstein-Barr Virus status and Its prognostic value in lymphoma. Ann Hematol Oncol. 2016;3:6.

6. Hatton OL, Harris-Arnold A, Schaffert S, Krams SM, Martinez OM. The interplay between Epstein-Barr virus and $B$ lymphocytes: implications for infection, immunity, and disease. Immunol Res. 2014;58:268-76.

7. Houldcroft CJ, Kellam P. Host genetics of Epstein-Barr virus infection, latency and disease. Rev Med Virol. 2015;25:71-84.

8. Manzo T, Heslop HE, Rooney CM. Antigen-specific T cell therapies for cancer. Hum Mol Genet. 2015;24:R67-73.

9. Merlo A, Turrini R, Dolcetti R, Martorelli D, Muraro E, Comoli P, et al. The interplay between EpsteinBarr virus and the immune system: a rationale for adoptive cell therapy of EBV-related disorders. Haematologica. 2010;95:1769-77.

10. Agathanggelou A, Niedobitek G, Chen R, Nicholls J, Yin W, Young LS. Expression of immune regulatory molecules in Epstein-Barr virus-associated nasopharyngeal carcinomas with prominent lymphoid stroma. Evidence for a functional interaction between epithelial tumor cells and infiltrating lymphoid cells. Am J Pathol. 1995;147:1152-60.

11. Huang YT, Sheen TS, Chen CL, Lu J, Chang Y, Chen JY, et al. Profile of cytokine expression in nasopharyngeal carcinomas: a distinct expression of interleukin 1 in tumor and CD4 + T cells. Cancer Res. 1999;59:1599-605.

12. Lau KM, Cheng SH, Lo KW, Lee SA, Woo JK, van Hasselt CA, et al. Increase in circulating Foxp3 + CD4 + CD25(high) regulatory T cells in nasopharyngeal carcinoma patients. Br J Cancer. 2007;96:61722.

13. Marshall NA, Vickers MA, Barker RN. Regulatory T cells secreting IL-10 dominate the immune response to EBV latent membrane protein 1. J Immunol. 2003;170:6183-9.

14. Crespo J, Sun H, Welling TH, Tian Z, Zou W. T cell anergy, exhaustion, senescence, and stemness in the tumor microenvironment. Curr Opin Immunol. 2013;25:214-21.

15. Kuklina EM. Molecular mechanisms of T-cell anergy. Biochemistry. 2013;78:144-56.

16. Kryczek I, Zou L, Rodriguez P, Zhu G, Wei S, Mottram P, et al. B7-H4 expression identifies a novel suppressive macrophage population in human ovarian carcinoma. J Exp Med. 2006;203:871-81.

17. Curiel TJ, Wei S, Dong H, Alvarez X, Cheng P, Mottram P, et al. Blockade of B7-H1 improves myeloid dendritic cell-mediated antitumor immunity. Nat Med. 2003;9:562-7.

18. Blank C, Kuball J, Voelkl S, Wiendl H, Becker B, Walter B, et al. Blockade of PD-L1 (B7-H1) augments human tumor-specific T cell responses in vitro. Int J Cancer. 2006;119:317-27. 
19. Dong H, Strome SE, Salomao DR, Tamura H, Hirano F, Flies DB, et al. Tumor-associated B7-H1 promotes T-cell apoptosis: a potential mechanism of immune evasion. Nat Med. 2002;8:793-800.

20. Nazareth MR, Broderick L, Simpson-Abelson MR, Kelleher RJ Jr, Yokota SJ, Bankert RB. Characterization of human lung tumor-associated fibroblasts and their ability to modulate the activation of tumor-associated T cells. J Immunol. 2007;178:5552-62.

21. Wherry EJ. T cell exhaustion. Nat Immunol. 2011;12:492-9.

22. Wherry EJ, Kurachi M. Molecular and cellular insights into T cell exhaustion. Nat Rev Immunol. 2015;15:486-99.

23. Zarour HM. Reversing T-cell Dysfunction and Exhaustion in Cancer. Clin Cancer Res. 2016;22:185664.

24. Thommen DS, Schumacher TN. T Cell Dysfunction in Cancer. Cancer Cell. 2018;33:547-62.

25. Shi L, Chen S, Yang L, Li Y. The role of PD-1 and PD-L1 in T-cell immune suppression in patients with hematological malignancies. J Hematol Oncol. 2013;6:74.

26. Riches JC, Davies JK, McClanahan F, Fatah R, Iqbal S, Agrawal S, et al. T cells from CLL patients exhibit features of T-cell exhaustion but retain capacity for cytokine production. Blood. 2013;121:1612-21.

27. Zelle-Rieser C, Thangavadivel S, Biedermann R, Brunner A, Stoitzner P, Willenbacher E, et al. T cells in multiple myeloma display features of exhaustion and senescence at the tumor site. J Hematol Oncol. 2016;9:116.

28. Linton P, Thoman ML. T cell senescence. Front Biosci. 2001;6:D248-61.

29. He R, Hou S, Liu C, Zhang A, Bai Q, Han M, et al. Follicular CXCR5- expressing CD8(+) T cells curtail chronic viral infection. Nature. 2016;537:412-28.

30. Francisco LM, Sage PT, Sharpe AH. The PD-1 pathway in tolerance and autoimmunity. Immunol Rev. 2010;236:219-42.

31. Green MR, Rodig S, Juszczynski P, Ouyang J, Sinha P, O'Donnell E, et al. Constitutive AP-1 activity and EBV infection induce PD-L1 in Hodgkin lymphomas and posttransplant lymphoproliferative disorders: implications for targeted therapy. Clin Cancer Res. 2012;18:1611-8.

32. Romano A, Conticello C, Cavalli M, Vetro C, La Fauci A, Parrinello NL, et al. Immunological dysregulation in multiple myeloma microenvironment. Biomed Res Int. 2014; 2014: 198539.

33. Ma SD, Xu X, Jones R, Delecluse HJ, Zumwalde NA, Sharma A, et al. PD-1/CTLA-4 Blockade Inhibits Epstein-Barr Virus-Induced Lymphoma Growth in a Cord Blood Humanized-Mouse Model. PLoS Pathog. 2016;12:e1005642.

34. Quan L, Chen X, Liu A, Zhang Y, Guo X, Yan S, et al. PD-1 Blockade Can Restore Functions of T-Cells in Epstein-Barr Virus-Positive Diffuse Large B-Cell Lymphoma In Vitro. PLoS One. 2015;10:e0136476.

35. Kao C, Oestreich KJ, Paley MA, Crawford A, Angelosanto JM, Ali MA, et al. Transcription factor T-bet represses expression of the inhibitory receptor PD-1 and sustains virus-specific CD $8+T$ cell responses during chronic infection. Nat Immunol. 2011;12:663-71. 
36. Paley MA, Kroy DC, Odorizzi PM, Johnnidis JB, Dolfi DV, Barnett BE, et al. Progenitor and terminal subsets of CD8 + T cells cooperate to contain chronic viral infection. Science. 2012;338:1220-5.

37. Ansell SM, Lesokhin AM, Borrello I, Halwani A, Scott EC, Gutierrez M, et al. PD-1 blockade with nivolumab in relapsed or refractory Hodgkin's lymphoma. N Engl J Med. 2015;372:311-9.

38. Hegel JK, Knieke K, Kolar P, Reiner SL, Brunner-Weinzierl MC. CD152 (CTLA-4) regulates effector functions of CD8 + T lymphocytes by repressing Eomesodermin. Eur J Immunol. 2009;39:883-93.

39. Effros RB. T cell replicative senescence: pleiotropic effects on human aging. Ann N Y Acad Sci. 2004;1019:123-6.

40. Suen H, Brown R, Yang S, Weatherburn C, Ho PJ, Woodland N, et al. Multiple myeloma causes clonal T-cell immunosenescence: identification of potential novel targets for promoting tumour immunity and implications for checkpoint blockade. Leukemia. 2016;30:1716-24.

41. Pauken KE, Wherry EJ. SnapShot: T Cell Exhaustion. Cell. 2015;163:1038-8. e1031.

42. Nikiforow S, Alyea EP. Maximizing GVL in allogeneic transplantation: role of donor lymphocyte infusions. Hematology Am Soc Hematol Educ Program. 2014; 2014: 570-575.

43. Bollard CM, Gottschalk S, Torrano V, Diouf O, Ku S, Hazrat Y, et al. Sustained complete responses in patients with lymphoma receiving autologous cytotoxic $T$ lymphocytes targeting Epstein-Barr virus latent membrane proteins. J Clin Oncol. 2014;32:798-808.

44. Bollard CM, Gottschalk S, Leen AM, Weiss H, Straathof KC, Carrum G, et al. Complete responses of relapsed lymphoma following genetic modification of tumor-antigen presenting cells and Tlymphocyte transfer. Blood. 2007;110:2838-45.

\section{Figures}

A

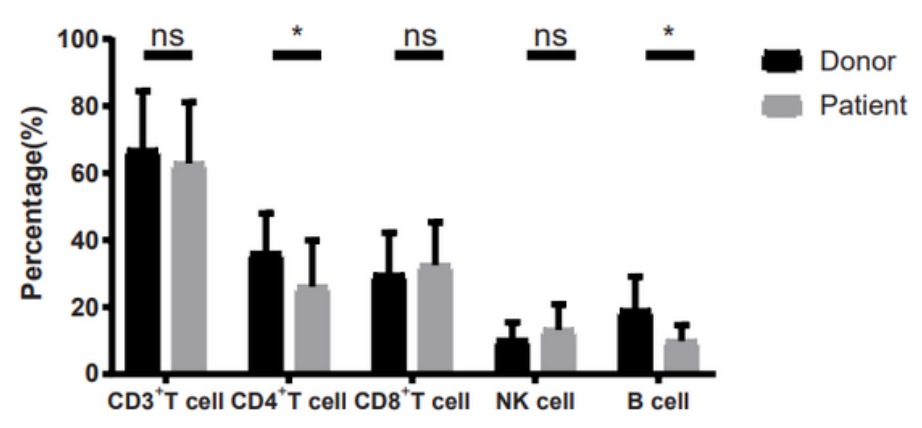

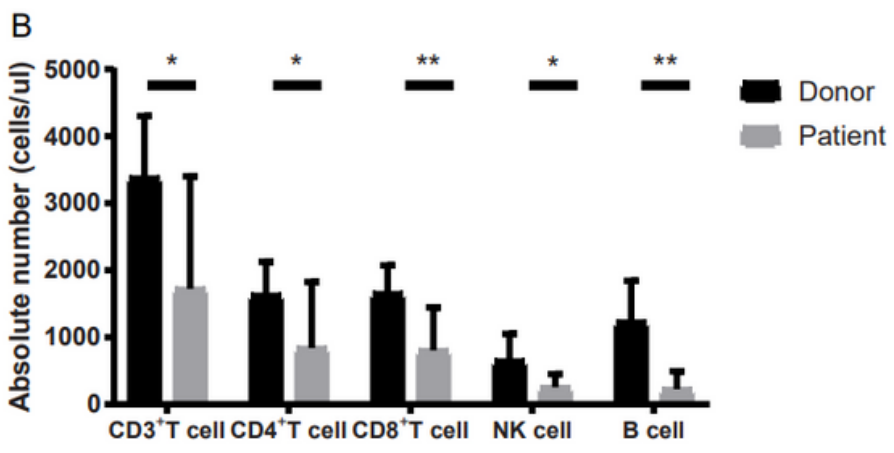

\section{Figure 1}

The proportions and absolute cell counts of lymphocyte subpopulations in EBV-associated lymphoma patients and healthy donors. A, The proportions of CD3+T cells (CD3+), CD4+ T cells (CD3+CD4+), CD8+ T cells (CD3+CD8+), NK cells (CD3-CD56+) and B cells (CD3-CD19+) gated on CD45+ cells in peripheral 
blood were shown. $B$, The absolute cell counts of CD3+T cells, CD4+ T cells, CD8+ T cells, NK cells and B cells per ul were shown. *: $p<0.05$; $* *$ : $p<0.01$; and ***: $p<0.001$

A

Donor 1

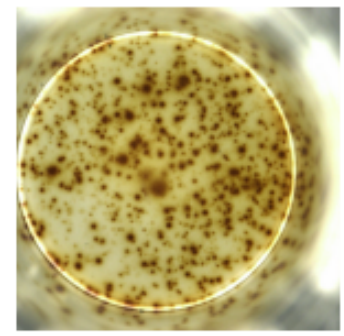

B

Patient 1

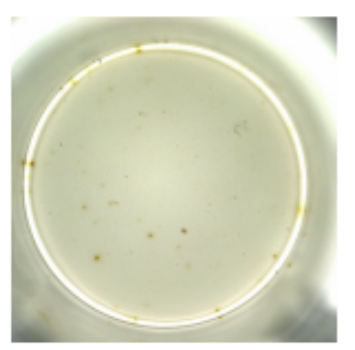

Donor 2

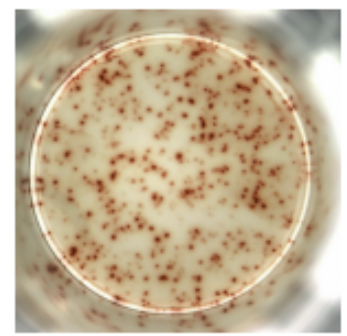

Patient 2

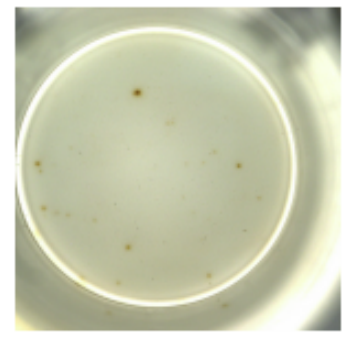

C

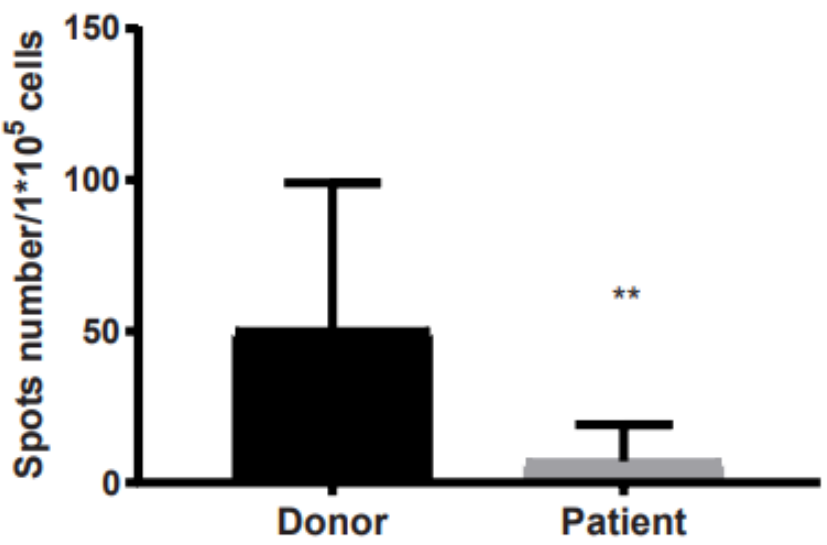

Figure 2

EBV antigen-specific cytotoxic T cells in EBV-associated lymphoma patients and healthy donors. After 24 hours stimulation with EBV mixed peptides, the production of IFN- $y$ of T cells in peripheral blood in two representative healthy donors $(A)$ and EBV-associated lymphoma patients(B) were shown. (C) The spot counts in healthy donors and lymphoma patients. **: $p<0.01$ 


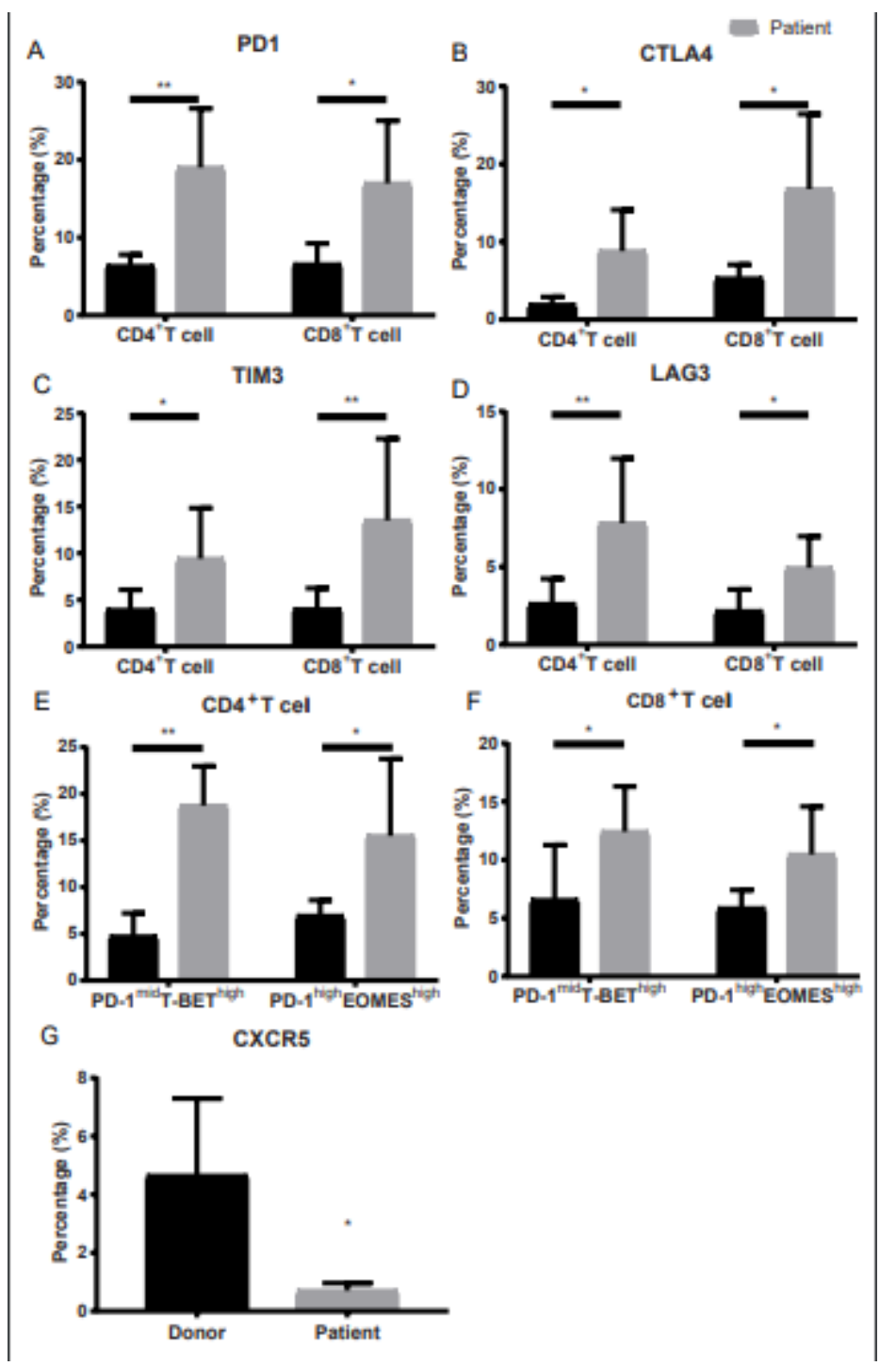

Figure 3

T cell exhaustion in patients with EBV-associated lymphoma and healthy donors. A-D, The percentages of PD1, CTLA4, TIM3 and LAG3 positive cells in CD4+ T cells and CD8+ T cells in peripheral blood of EBVassociated lymphoma patients and healthy donors respectively. E-F, The proportions of two exhausted phenotypes gated on $\mathrm{CD} 4+\mathrm{T}$ and $\mathrm{CD} 8+\mathrm{T}$ cells in peripheral blood of patients with EBV-associated lymphoma and healthy donors. G, The percentages of CXCR5 positive cells in CD8+ T cells in EBVassociated lymphoma patients and healthy donors. *: $p<.05 ; * *: p<.01$.
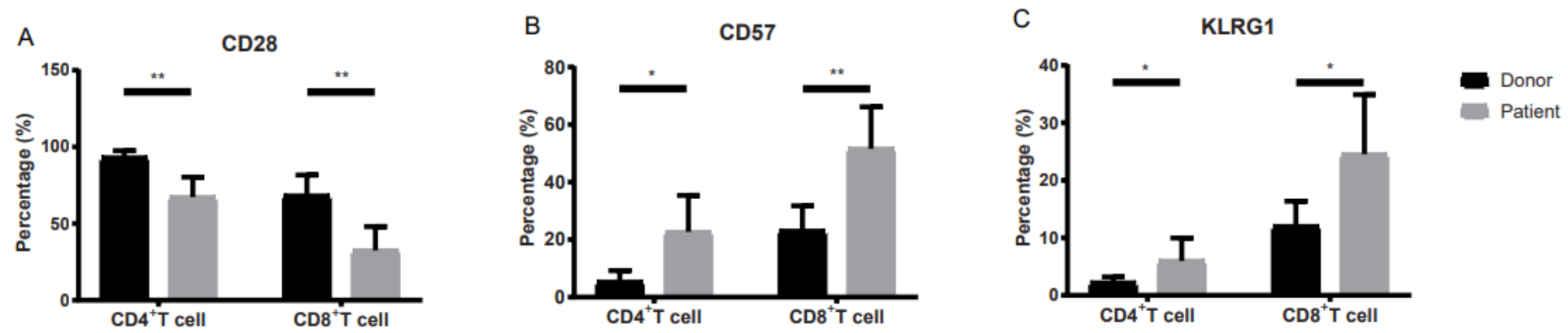


\section{Figure 4}

T cell senescence in patients with EBV-associated lymphoma and healthy donors. Percentage of CD28 (A), CD57 (B) and KLRG-1 (C) positive cells in CD4+ and CD8+ T cells in peripheral blood of patients with EBV-associated lymphoma and healthy donors. $*$ : $p<.05 ; * \star$ : $p<.01$. 